\title{
Importância Prognóstica da Invasão Neural no Câncer Colorretal: Estudo Imunoistoquímico com a Proteína S-100
}

\author{
Prognostic Importance of Neural Invasion in Colorectal Cancer: \\ An Immunohistochemical Study With the S-100 Protein
}

\author{
JOSÉ VINIICIUS CRUZ ; RAULCUTAIT ${ }^{3}$; ${\text { CARLOS AUGUSTO REAL MARTINEZ }{ }^{4} \text {; RUY GERALDO BEVILACQUA }}^{3}$; \\ KÁTIARAMOS MOREIRALEITE ${ }^{5}$ NELSON FONTANAMARGARIDO ${ }^{6}$
}

\begin{abstract}
${ }^{2}$ Professor Titular Doutor da Disciplina de Coloproctologia da Faculdade Federal de Ciências Médicas de Porto Alegre, RS.Brasil; ${ }^{3}$ Professor Associado do Departamento de Cirurgia da Faculdade de Medicina da Universidade de São Paulo, SP.Brasil; ${ }^{4}$ Professor Adjunto Doutor do Programa de Pós-Graduação em Ciências da Saúde da Universidade São Francisco, Bragança Paulista, SP.Brasil; ${ }^{5}$ Médica do Serviço de Anatomia Patológica do Hospital Sírio Libanês, São Paulo, SP.Brasil; ${ }^{6}$ Professor Associado do Departamento de Cirurgia da Faculdade de Medicina da Universidade de São Paulo. Professor Responsável pela Disciplina de Técnica Cirúrgica da Faculdade de Medicina da Universidade de São Paulo, SP - Brasil.
\end{abstract}

CRUZ JV; CUTAIT R; MARTINEZ CAR; BEVILACQUA RG; LEITE KRM; MARGARIDO NF. Importância Prognóstica da Invasão Neural no Câncer Colorretal:Estudo Imunoistoquímico com a Proteína S-100. Rev bras Coloproct, 2006;26(3): 280-292.

\begin{abstract}
RESUMO: Das variáveis anatomopatológicas relacionadas ao prognóstico de enfermos com câncer colorretal, a invasão neural ainda se encontra pouco estudada. Objetivo: Verificar se a invasão neural no câncer colorretal estádios B e C de Dukes pode ser considerada como fator prognóstico independente. Método: Foram estudados 97 doentes operados com intenção curativa e seguidos por período mínimo de cinco anos. Excluíram-se doentes que receberam tratamento adjuvante. Os espécimes cirúrgicos foram corados por hematoxilina-eosina e imunoistoquímica para pesquisa da proteína $\mathrm{S}-100$, com o intuito de se comparar a fidedignidade das técnicas em detectar invasão neural, sendo analisadas comparativamente: acurácia, especificidade, sensibilidade e valores preditivos positivo e negativo. A comparação entre a incidência de invasão neural com relação à recidiva foi realizada, empregando-se o teste do qui-quadrado. A sobrevida e sobrevida livre de doença foram estudadas por análise univariada,. Estabeleceu-se nível de significância de $5 \%$ (p $£$ 0,05) para todos os testes adotados. Resultados: A técnica da HE apresentou fraca habilidade em detectar a invasão neural, não sendo adequada para esta análise em doentes portadores de câncer colorretal. As curvas de sobrevida e sobrevida livre de doença dos enfermos portadores de invasão neural, pesquisada por meio da imunocoloração para proteína S-100 são significativamente piores, identificando aquela característica histológica como valor prognóstico independente ( $p=0,0003$ e $p=0,0002$, respectivamente). A ocorrência de recidiva tumoral foi significativamente maior nos doentes que apresentavam invasão neural $(p=0,0010)$. Conclusão: Os resultados do presente estudo permitem concluir que, nos doentes portadores de câncer colorretal, a detecção da invasão neural pela pesquisa imunoistoquímica da proteína $S$-100 demonstrou ser variável independente, acrescentando informações prognósticas adicionais nos doentes classificados nos estádios B, C e C2 das classificações de Dukes e Astler-Coller, respectivamente.
\end{abstract}

Descritores: Neoplasias. Neoplasias Colorretais. Proteína S 100. Imunohistoquímica. Prognóstico.

\footnotetext{
${ }^{1}$ Trabalho realizado pelo Serviço de Coloproctologia do Hospital Sírio Libanês, São Paulo e pela Disciplina de Técnica Cirúrgica da Faculdade de Medicina da Universidade de São Paulo.Brasil.
}

Recebido em 10/05/2006

Aceito para publicação em 01/08/2006 


\section{INTRODUÇÃO}

Nos Estados Unidos, o câncer colorretal (CCR) representa a segunda causa de morte por neoplasia entre os homens e a terceira entre as mulheres ${ }^{1}$. Os índices de mortalidade vêm se alterando lentamente, não obstante os recentes avanços obtidos no diagnóstico precoce e tratamento ${ }^{2}$. No Brasil, a evolução do CCR tem apresentado comportamento similar, sendo, atualmente, a quinta causa mais comum de morte relacionada ao câncer e, à semelhança de outros países, sua incidência vem aumentando em comparação a outros tipos de tumores que acometem o aparelho digestivo $^{3,4}$.

A variabilidade dos comportamentos clínico e biológico do CCR tem suscitado grande interesse pelo estudo de fatores que possam estar associados à progressão da neoplasia e, conseqüentemente, ao prognóstico da doença ${ }^{5,6}$. O conhecimento do maior número de variáveis diretamente relacionadas ao prognóstico da enfermidade torna-se necessário com o objetivo de tentar identificar os doentes que realmente poderão se beneficiar do tratamento complementar. Estudos tentando correlacionar variáveis clínicas, biológicas, histopatológicas e moleculares ao prognóstico do CCR, têm sido publicados, mas seus resultados são intrigantes e muitas vezes conflituosos ${ }^{7-9}$. Assim, o diagnóstico precoce, ${ }^{10}$ a idade, ${ }^{11}$ localização da neoplasia, ${ }^{12}$ graduação histológica, ${ }^{13}$ grau de penetração na parede intestinal, ${ }^{14}$ comprometimento linfonodal, ${ }^{15,16}$ produção de muco pela neoplasia, ${ }^{17}$ antígeno carcinoembrionário, ${ }^{18,19} \mathrm{e}$ a invasão venosa e neural, ${ }^{20,21}$ são as variáveis mais freqüentemente estudadas, como fatores relacionados ao prognóstico da doença. Apesar da importância de todos esses fatores, o comprometimento parietal e linfonodal continuam representando a principal base de predição da sobrevida e critério para instituição da terapia adjuvante ${ }^{14}$.

Numerosas tentativas foram realizadas com o intuito de discriminar o poder prognóstico independente das principais variáveis relacionadas ao $\mathrm{CCR}^{22,23}$. O consenso dessas publicações revela que os fatores que apresentam associações mais fortes com a evolução dos doentes são: profundidade de infiltração do tumor na parede intestinal, o comprometimento linfonodal, a presença de metástases e a graduação histológica da neoplasia ${ }^{22,23}$. Entretanto, as tentativas de avaliação prognóstica apresentam a grave limita- ção de restringirem-se à observação de um registro microscópico momentâneo da morfologia tumoral, não fornecendo informações sobre o real comportamento biológico do tumor, o qual se encontra relacionado aos mecanismos intrínsecos de funcionamento das células componentes daquele tecido ${ }^{22}$. Recentemente, com a melhor compreensão das alterações genéticas envolvidas na carcinogênese colorretal, a pesquisa de instabilidades de cromossomos e genes, bem como as alterações da expressão tecidual de proteínas por eles codificadas vêm tornando atraente a possibilidade do emprego de marcadores moleculares como variáveis potencialmente válidas para melhor compreensão do prognóstico do CCR. ${ }^{23,24}$ Com o advento dos métodos de análise indireta da expressão de genes, pela detecção tecidual das proteínas por eles codificadas com técnicas imunoistoquímicas, uma nova dimensão no estudo da carcinogênese colorretal foi conquistada. A possibilidade de detecção tecidual de antígenos relacionados ao desenvolvimento, crescimento e disseminação do tumor permite avaliar a importância deles como fatores relacionados ao prognóstico da enfermidade ${ }^{23,24}$.

Analogamente à invasão angiolinfática, considerada importante fator prognóstico nos portadores de CCR, a invasão neural (IN) também tem sido apontada como potencial fator preditivo. Estima-se que a invasão da bainha nervosa por células neoplásicas possa ocorrer em 14 a $32 \%$ dos doentes, quando avaliada por meio de técnicas histológicas convencionais, estando associada a pior prognóstico ${ }^{25-27}$. Contudo, a detecção da IN pelas técnicas de coloração convencionais é de difícil interpretação, principalmente em áreas onde o crescimento neoplásico, o infiltrado inflamatório, a necrose e a fibrose peritumoral alteraram a arquitetura da parede intestinal ${ }^{28}$. O emprego de técnicas imunoistoquímicas possibilitou maior precisão na avaliação do comprometimento nervoso. Estudos comparativos entre técnicas convencionais e imunoistoquímicas para o diagnóstico da IN encontraram índices diagnósticos de $14 \%$ e $70 \%$ respectivamente, aumentando consideravelmente a precisão diagnóstica, fazendo com que a UICC (International Union Against Cancer) passasse a considerá-la como provável fator prognóstico ${ }^{29}$.

No estudo da IN em doentes portadores de câncer do aparelho digestivo, a pesquisa da expressão tecidual da proteína S-100, por técnica imunoistoquímica 
vêm apresentando resultados promissores ${ }^{29,30}$. Todavia, foram poucos os estudos que avaliaram a expressão tecidual da proteína S-100, por meio de imunoistoquímica, como metodologia para analisar com maior precisão, a real incidência do comprometimento nervoso nos doentes operados por CCR, relacionandoa ao prognóstico da doença.

O objetivo do presente estudo foi avaliar a IN por meio da expressão tecidual da proteína $\mathrm{S}-100 \mathrm{em}$ doentes portadores de CCR estádios B e C de Dukes e verificar sua relação com aspectos prognósticos na casuística examinada.

\section{MÉTODO}

Após aprovação pelo Comitê de Ética do Hospital Sírio Libanês, foram analisados espécimes cirúrgicos com adenocarcinoma colorretal, incluídos em blocos de parafina, de 122 doentes operados entre 1977 e 1990, por um único cirurgião (RC). Incluiram-se no estudo todos os enfermos operados com intenção curativa que não apresentavam metástases (exceto linfonodais), avaliados durante estadiamento pré e intraoperatório e seguidos por período mínimo de cinco anos ou até a recidiva da neoplasia. Foram excluídos 25 casos, sendo cinco por seguimento incompleto, 17 por doença metastática ou previamente submetidos a tratamento adjuvante no pré ou pós-operatório, e três pacientes classificados como estádio A de Dukes, por não constituírem subgrupo representativo para análise. Dessa forma, a amostra ficou restrita a 97 doentes (47 mulheres), com idades variando entre 25 e 84 anos, portadores de adenocarcinoma do cólon e reto, classificados nos estádios B e C de Dukes. Apesar da classificação de Dukes restringir-se aos tumores de reto, no presente estudo foi também estendida às neoplasias do cólon, à semelhança de trabalhos anteriores ${ }^{18,19}$.

As principais variáveis clínicas e histológicas estudadas foram: idade (maior ou menor que 60 anos), sexo, localização do tumor (reto ou cólon), profundidade de invasão da parede cólica (Dukes e Astler-Coller), grau histológico (pouco, moderadamente ou bem diferenciado), tipo histológico (tubular, papilífero, mucinoso, células em anel de sinete), forma de crescimento tumoral (expansivo ou infiltrativo), linfonodos (número de linfonodos ressecados e comprometidos), infiltrado inflamatório (discreto, moderado, extenso), fibrose (discreta, moderada, extensa), necrose (ausente, focal, difusa), evolução clínica (favorável ou desfavorável), localização da recidiva (loco-regional ou à distância), seguimento (em meses desde a cirurgia até a última revisão clínica), tempo livre de doença (em meses desde a cirurgia até a identificação da recidiva), tempo de sobrevida em meses (desde a cirurgia até o óbito pela doença).

As lâminas foram confeccionadas em um único laboratório e analisadas por patologista experiente em neoplasias digestivas e em técnicas imunoistoquímicas. Na análise histológica, empregaramse dois métodos de coloração: hematoxilina-eosina não ácida e imunoistoquímico para pesquisa da proteína S-100, utilizando-se com anticorpo primário antiproteína S-100 (Dako CytomationÒ, Copenhagen, Dinamarca) com a técnica do complexo avidina-biotina-peroxidase (ABC-VectorÒ), segundo metodologia anteriormente descrita. ${ }^{19}$

Para a análise de aspectos anatomopatológicos adotou-se a classificação proposta por Jass e Morson ${ }^{31}$, estabelecendo-se escala de valores com pontuação específica, classificando os doentes com CCR em quatro classes de gravidade crescente, considerando-se: grau de penetração do tumor na parede intestinal, tipo de crescimento tumoral (expansivo ou infiltrativo), número de linfonodos comprometidos e infiltrado inflamatório (extenso ou discreto). Acrescentou-se à classificação a presença ou não de IN pesquisada pela proteína S-100.

$\mathrm{Na}$ análise estatística, a fidedignidade da coloração pela hematoxilina-eosina para confirmação da IN, em relação ao método imunoistoquímico para pesquisa da proteína S-100, foi analisada quanto a acurácia, sensibilidade, especificidade, valor preditivo positivo e negativo. A análise das variáveis anatomopatológicas e sobrevida foi feita pela apresentação das frequiências e a porcentagem dos subtotais de sobrevida em $5 \mathrm{e}$ 10 anos, nas condições de invasão neural presente ou ausente.

Para análise univariada, foram calculadas as curvas de Kaplan-Meier para os tempos de sobrevida e sobrevida livre para cada variável considerada. A significância das diferenças entre as curvas foi avaliada pelos testes de Cox-Mantel (Log-Rank) e Breslow. A partir das variáveis que se mostraram estatisticamente significativas na análise univariada, realizou-se a analise de regressão multivariada pelo método de Cox. Utilizou-se o modelo passo a passo, inclusivo e 
condicional, tendo como critério de inclusão $\mathrm{p}<0,05$ e de exclusão $p>0,10$. Como todas as variáveis consideradas eram categóricas, elas foram codificadas por contraste simples. Cada variável foi comparada à categoria que se mostrou de melhor prognóstico na análise univariada. $\mathrm{Na}$ análise da recidiva tumoral com a condição de IN pelo tumor, considerando o caráter qualitativo das variáveis correlacionadas, os resultados foram avaliados pelo teste do $\mathrm{c}^{2}$. Para todos os testes utilizados adotou-se nível crítico de $5 \%(\mathrm{p}<0,05)$, empregando-se o programa SPSS-13.

\section{RESULTADOS}

O Quadro 1 mostra a distribuição dos pacientes segundo a localização do tumor, classificação de Dukes e IN.
A Figura 1 mostra, em um mesmo espécime, a comparação entre os dois métodos de coloração. Verifica-se que, enquanto o método convencional sugere a IN, a pesquisa da proteína S-100 mostra a preservação do nervo, tratando-se de resultado falso positivo.

A Figura 2 mostra a comparação em um mesmo espécime entre as colorações pela hematoxilinaeosina e a imunoistoquímica para proteína S-100. Podese verificar que, enquanto a técnica convencional sugere a não existência de comprometimento neural, a imunoistoquímica confirma a IN, tratando-se de resultado falso negativo.

O Quadro 2 mostra comparativamente, a fidedignidade do método da Hematoxilina-eosina quando comparado à pesquisa imunoistoquímica da proteína S100 no diagnóstico da IN.

Quadro 1 - Distribuição dos doentes de acordo com a localização do tumor, classificação de Dukes $e$ invasão neural.

\begin{tabular}{lccc}
\hline Localização & Classificação de Dukes & Invasão neural & n \\
\hline RETO & $\mathrm{B}$ & Presente & 9 \\
& & Ausente & 22 \\
& $\mathrm{C}$ & Presente & 15 \\
& & Ausente & 10 \\
CÓLON & $\mathrm{B}$ & Presente & 12 \\
& & Ausente & 14 \\
& $\mathrm{C}$ & Presente & 7 \\
& & Ausente & 8 \\
\hline
\end{tabular}

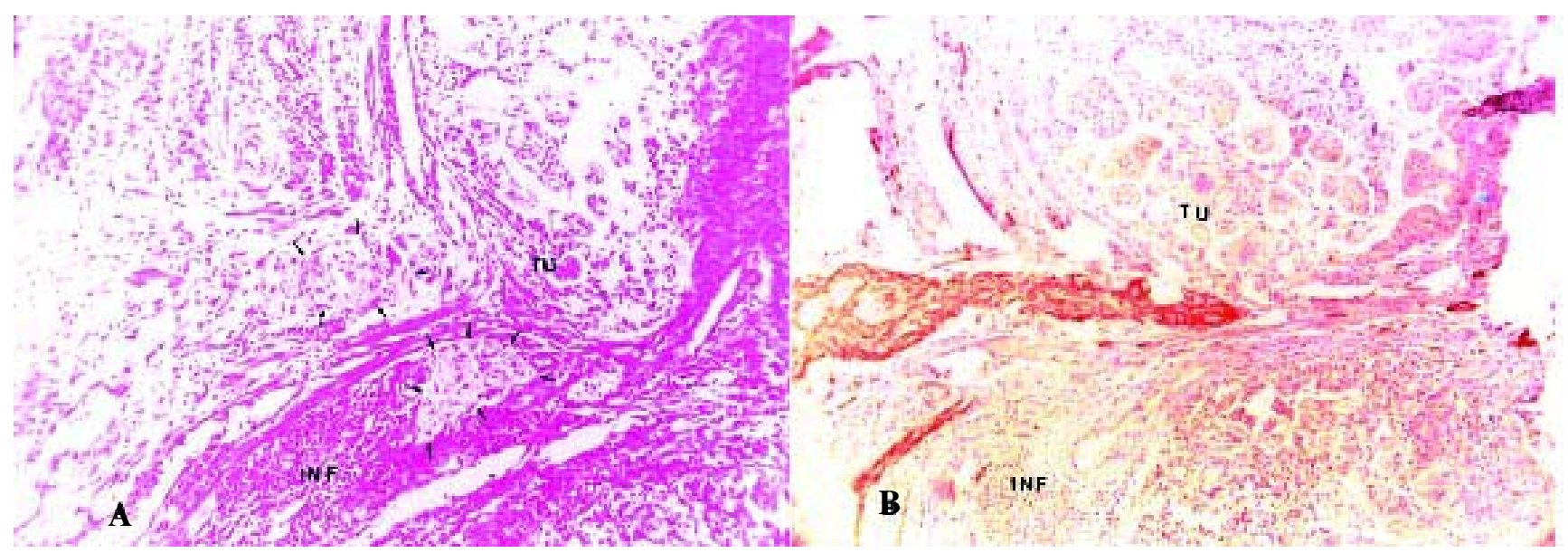

Figura 1-A-Intestino grosso com câncer, corado pela hematoxilina-eosina. Identifica-se tumor muco-secretor (TU), infiltrado inflamatório (INF), e as setas indicam o nervo aparentemente invadido pelo tumor. FALSO POSITIVO PARA INVASÃO NEURAL (40x). B-Mesmo espécime, corado pela imunoistoquímica com a proteína $S-100$ observando-se o tumor (TU), infiltrado inflamatório (INF) e a exata preservação do nervo $(40 x)$. 

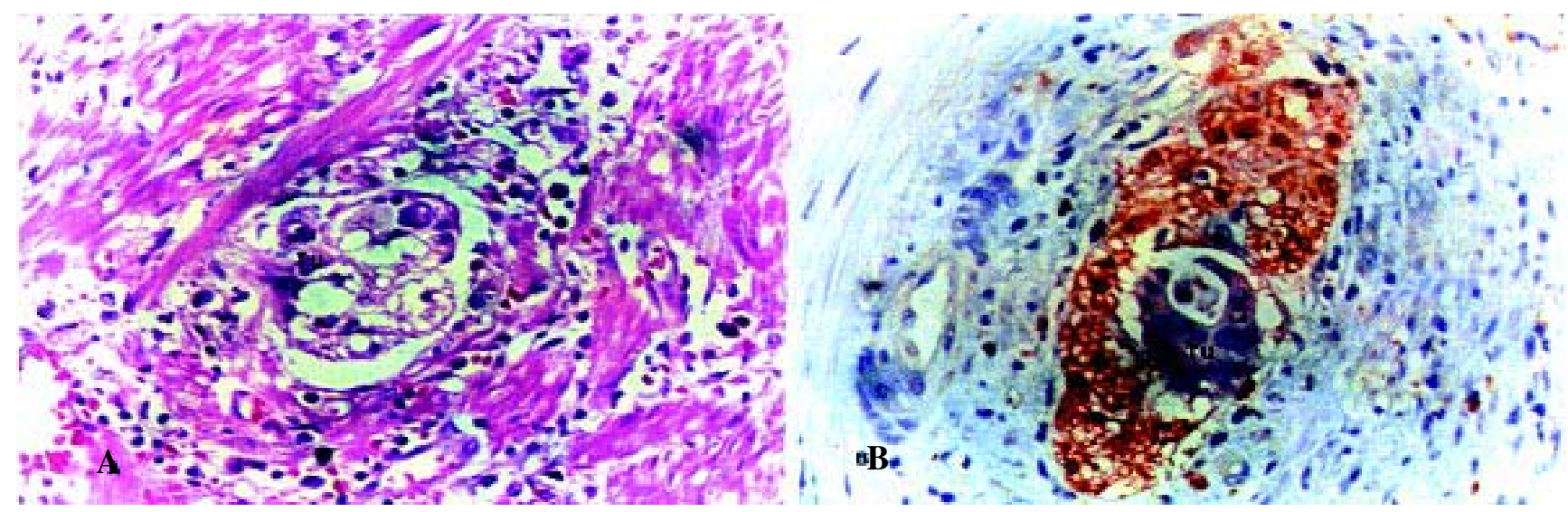

Figura 2-A-Intestino grosso com câncer, corado pela hematoxilina-eosina. Identifica-se tumor (TU), sem a aparente ocorrência de invasão neural. FALSO NEGATIVO PARA INVASÃ O NEURAL (100x). B- Mesmo corte de tecido, corado pela imunoistoquímica com a proteína S-100. O nervo corado em marrom apresenta nítida invasão tumoral. INVASÃO NEURAL POSITIVA (100x).

Quadro 2 - Número de casos com e sem invasão neural pelos dois métodos de coloração.

\begin{tabular}{lccc}
\hline \multirow{2}{*}{ CONDIÇÃo } & \multicolumn{3}{c}{ Método de Coloração } \\
\cline { 2 - 4 } & $\begin{array}{c}\text { Proteína S-100 } \\
\text { n }\end{array}$ & \multicolumn{2}{c}{ Hematoxilina- Eosina } \\
noM invasão neural & 43 & 22 & 21 \\
SEM invasão neural & & (verdadeiramente positivos) & (falsos negativos) \\
& 54 & 44 & 10 \\
& & (verdadeiramente negativos) & (falsos positivos) \\
\hline
\end{tabular}

A validade da coloração pela hematoxilinaeosina para confirmação da IN, em relação ao método imunoistoquímico para pesquisa da proteína S-100, demonstrou acurácia de $68 \%$, sensibilidade de $49 \%$, especificidade de $81 \%$, valor preditivo positivo de $69 \%$ e valor preditivo negativo de $69 \%$. Tais resultados comprometem a validade do método da hematoxilina-eosina em avaliar corretamente a IN.

A Tabela 1 mostra as variáveis estudadas que apresentaram significância estatística, os aspectos, o tempo médio de sobrevida (meses) em cinco anos, e a significância estatística por meio da análise univariada.

A Tabela 2 mostra as variáveis estudadas que apresentaram significância estatística, os aspectos, o tempo médio de sobrevida livre da doença (meses) em cinco anos, e a significância estatística por meio da análise univariada.

Ao se considerar as curvas de sobrevida, aplicando-se o teste de Cox-Mantel verificou-se que havia diferença significante, comparando-se os doentes com invasão neural positiva e negativa, nos doentes estádios B e C de Dukes (Figuras 3 e 4).

Ao se considerar as curvas de sobrevida livre de doença, verificou-se que não havia diferenças significantes nos doentes pertencentes aos estádios B1 e B2 de Astler-Coller. Quando se considerou a sobrevida livre de doença, verificou-se que as diferenças foram estatisticamente significantes nos doentes com estádio C1 e C2 da classificação de Astler-Coller. (Figuras 5 e 6 )

Com base nos critérios de Jass e Morson (número de pacientes, casos de óbito pela doença, sobrevida com e sem evidência da doença, óbitos por outras causas e a sobrevida em cinco e 10 anos), acrescentando-se apenas a separação de acordo com a presença ou ausência de IN, a mesma metodologia proposta pelos autores foi reproduzida. Os resultados dessa análise encontram-se reproduzidos na Tabela 3.

A partir dos testes de Cox-Mantel e Breslow, selecionaram-se as categorias de variáveis que se 
Tabela 1 - Variáveis significantes, aspectos, tempo médio de sobrevida em cinco anos e resultados da análise univariada.

\begin{tabular}{|c|c|c|c|c|}
\hline Variável & Aspecto & $\begin{array}{c}\text { Sobrevida em } 5 \text { anos } \\
(\text { meses } \pm \text { I.C. } 95 \%)\end{array}$ & Log-Rank & Breslow \\
\hline \multirow[t]{2}{*}{ Dukes } & B & $84 \pm 4$ & & \\
\hline & $\mathrm{C}$ & $69 \pm 9$ & $0,0064 *$ & $0,0140 *$ \\
\hline \multirow{4}{*}{ Astler Coller } & B1 & $86 \pm 6$ & & \\
\hline & B2 & $90 \pm 6$ & & \\
\hline & $\mathrm{C} 1$ & $70 \pm 8$ & $0,0092^{*}$ & $0,0299 *$ \\
\hline & $\mathrm{C} 2$ & $50 \pm 20$ & & \\
\hline \multirow[t]{4}{*}{ Tipo de tumor } & Papilífero & $70 \pm 12$ & & \\
\hline & Tubular & $84 \pm 4$ & & \\
\hline & Mucinoso & $57 \pm 19$ & $0,0000^{*}$ & $0,0000^{*}$ \\
\hline & Anel de sinete & $0 \pm 0$ & & \\
\hline \multirow[t]{2}{*}{ Invasão neural (S-100) } & Positiva & $59 \pm 8$ & & \\
\hline & Negativa & $94 \pm 3$ & $0,0000^{*}$ & $0,0000 *$ \\
\hline \multirow{2}{*}{$\mathrm{N}^{\mathrm{o}}$ de linfonodos comprometidos } & $<3$ & $83 \pm 4$ & & \\
\hline & $=\mathrm{ou}>3$ & $68 \pm 9$ & $0,0196^{*}$ & $0,0335^{*}$ \\
\hline \multirow[t]{2}{*}{$\%$ de linfonodos comprometidos } & $<20 \%$ & $84 \pm 4$ & & \\
\hline & $=$ ou $>20 \%$ & $65 \pm 10$ & $0,0217 *$ & $0,0335^{*}$ \\
\hline
\end{tabular}

mostraram de melhor prognóstico. O resultado da análise multivariada de fatores prognósticos para sobrevida, pelo método de Cox demonstrou que a classificação de Dukes $(\mathrm{p}=0,0169)$ e a IN, quando analisadas pela técnica imunoistoquímica ( $\mathrm{p}=0,0003)$, revelaram-se fatores prognósticos independentes.
De modo semelhante ao se analisar o resultado da análise multivariada dos fatores prognósticos para a sobrevida livre de doença, foi possível verificar que a IN analisada pela pesquisa imunoistoquímica da proteína $\mathrm{S}-100(\mathrm{p}=0,0002)$ também se revelou fator prognóstico independente.

Tabela 2 - Variáveis significantes, aspectos, tempo médio de sobrevida livre da doença e resultados da análise univariada.

\begin{tabular}{llccc}
\hline Variável & Aspecto & $\begin{array}{c}\text { Sobrevida em 5 anos } \\
\text { (meses } \pm \text { I.C. 95\%) }\end{array}$ & Log-Rank & Breslow \\
\hline Sexo & Masculino & $68 \pm 7$ & & \\
& Feminino & $42 \pm 7$ & $0,0249^{*}$ & 0,0683 \\
Dukes & B & $61 \pm 6$ & & \\
& $\mathrm{C}$ & $48 \pm 8$ & $0,0289^{* *}$ & $0,0220^{*}$ \\
Astler-Coller & $\mathrm{B} 1$ & $56 \pm 8$ & & \\
& $\mathrm{~B} 2$ & $68 \pm 10$ & & \\
& $\mathrm{C} 1$ & $53 \pm 8$ & $0,0154^{*}$ & $0,0158^{*}$ \\
& $\mathrm{C} 2$ & $17 \pm 15$ & & \\
Invasão neural (S-100) & Positiva & $39 \pm 7$ & & \\
& Negativa & $68 \pm 6$ & $0,0002^{*}$ & $0,0001^{*}$ \\
Porcentagem de linfonodos comprometidos & $<20 \%$ & $59 \pm 6$ & & \\
& $=$ ou > 20\% & $46 \pm 10$ & 0,0612 & $0,0324^{*}$ \\
\hline
\end{tabular}




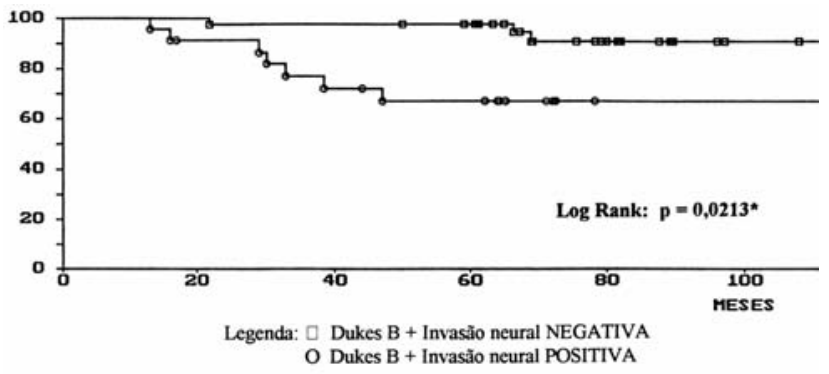

Figura 3 - Sobrevida relacionada a Dukes B e invasão neural pela pesquisa imunoistoquímica da proteína $S-100$.

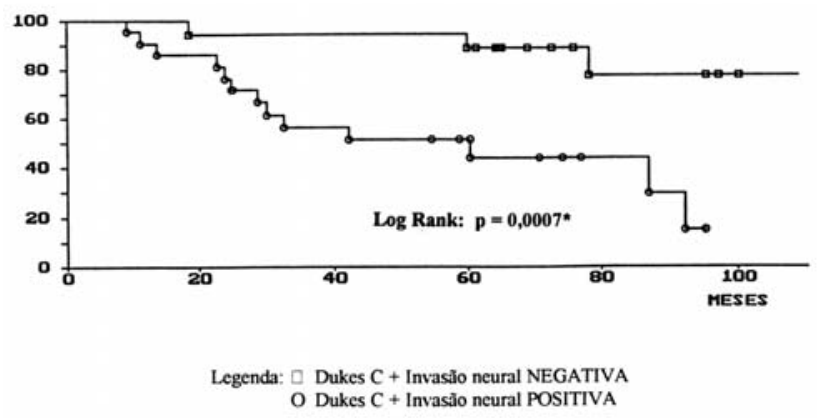

Figura 4 - Sobrevida relacionada a Dukes $C$ e invasão neural pela pesquisa imunoistoquímica da proteína $S-100$.

A Tabela 4 mostra os resultados da ocorrência de recidiva da doença tumoral, como um todo, independente do local, contra a presença ou não da IN pela neoplasia.

\section{DISCUSSÃO}

O conhecimento do maior número de variáveis diretamente relacionadas ao prognóstico do CCR torna-se necessário com o objetivo de se identificar mais precisamente os doentes que realmente poderão se beneficiar do emprego do tratamento complemen$\operatorname{tar}^{5,6}$. A ressecção cirúrgica continua sendo a mais efetiva modalidade terapêutica para o $\mathrm{CCR}^{32}$. A cura e prazos mais longos de sobrevida podem ser alcançados somente nos doentes submetidos à cirurgia com caráter curativo. Exceções são os poucos enfermos com CCR em estágio precoce nos quais a destruição tumoral pode ser alcançada somente por radio ou radioquimioterapia. Nos pacientes com doença residual conhecida, o prognóstico é relativamente uniforme e influenciado por limitado número de fatores e, em conseqüência, o reconhecimento deles terá pouca ou nenhuma influência nas ações terapêuticas. Em contraste, nos enfermos em que se realizou a ressecção

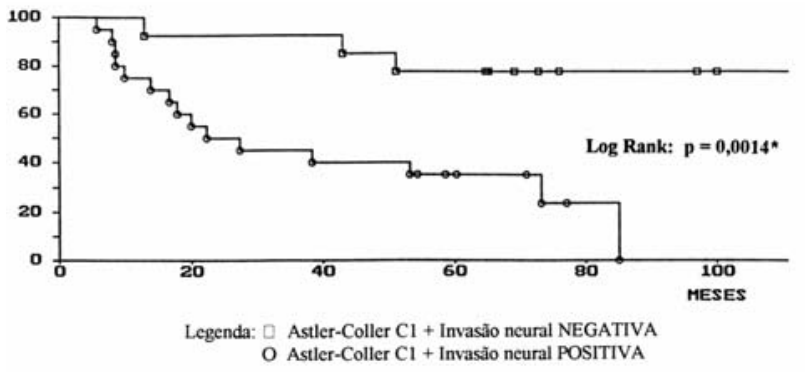

Figura 5 - Sobrevida livre de doença relacionada a Astler-Coller C1 e invasão neural pela pesquisa imunoistoquímica da proteína $S-100$.

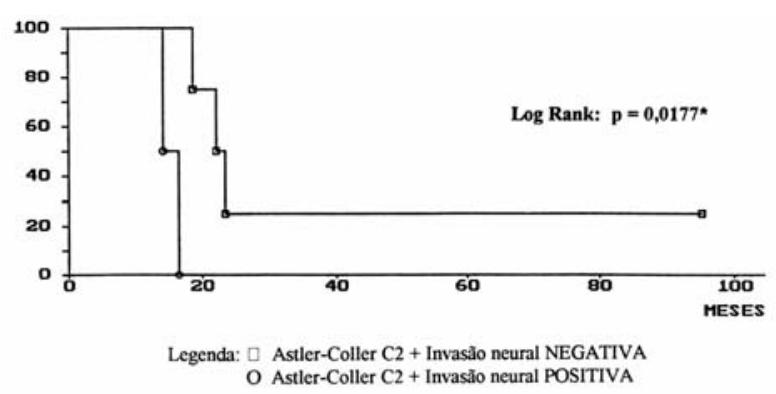

Figura 6-Sobrevida livre de doença relacionada a Astler-Coller C2 e invasão neural pela pesquisa imunoistoquímica da proteína S-100.

completa do tumor, existe amplo espectro relativo à sua evolução clínica. Neste grupo, diversos fatores influenciam o prognóstico, tornando-se fundamental definir os doentes de alto risco nos quais tratamentos adjuvantes e neoadjuvantes poderiam ser considerados.

Os fatores prognósticos servem para muitos propósitos. São utilizados para melhor compreensão da história natural do câncer, para a predição dos resultados dos procedimentos terapêuticos, para identificar grupos homogêneos de enfermos, para comparar resultados de diferentes tratamentos, para identificar grupos de doentes com evolução desfavorável e no planejamento de estratégias de seguimento clínico-cirúrgico ${ }^{33}$. A utilização de fatores prognósticos também permite a individualização de tratamentos, reservando a estratégia mais agressiva para doentes com variáveis mais adversas e o tratamento menos agressivo para aqueles com fatores prognósticos favoráveis. A melhoria da acurácia prognóstica poderia também contribuir para o alívio das incertezas dos portadores de CCR, melhorando aspectos humanos e psicológicos relacionados aos cuidados médicos dispensados.

Os fatores prognósticos podem ser divididos em três grupos distintos: os relacionados ao doente, os 
Tabela 3 - Resultados da aplicação da metodologia de Jass e Morson (1987) quanto a variáveis anatomopatológicas, óbitos e sobrevida dos pacientes com e sem invasão neural.

\begin{tabular}{|c|c|c|c|c|c|c|c|c|}
\hline \multirow[t]{2}{*}{ Grupos } & \multirow[t]{2}{*}{$\begin{array}{c}\text { Invasão } \\
\text { neural }\end{array}$} & \multirow[t]{2}{*}{$\mathbf{n}$} & \multirow[t]{2}{*}{$\begin{array}{c}\text { Óbitos } \\
\text { pela doença }\end{array}$} & \multicolumn{2}{|c|}{$\begin{array}{l}\text { SOBREVIDA } \\
\text { n }\end{array}$} & \multirow{2}{*}{$\begin{array}{l}\text { Óbitos por } \\
\text { outras causas } \\
\text { a }\end{array}$} & \multicolumn{2}{|c|}{$\begin{array}{c}\text { SOBREVIDA } \\
(\%) \\
\end{array}$} \\
\hline & & & & $\begin{array}{c}\text { Com } \\
\text { doença }\end{array}$ & $\begin{array}{c}\text { Sem } \\
\text { doença }\end{array}$ & & 5 anos & 10 anos \\
\hline \multirow[t]{2}{*}{ I } & Presente & 16 & 5 & 2 & 9 & - & 7 & 5 \\
\hline & Ausente & 26 & 3 & 5 & 15 & 3 & 15 & 10 \\
\hline \multirow[t]{2}{*}{ II } & Presente & 8 & 3 & 2 & 2 & 1 & 5 & - \\
\hline & Ausente & 10 & 1 & 1 & 7 & 1 & 8 & 2 \\
\hline \multirow[t]{2}{*}{ III } & Presente & 4 & 2 & 1 & - & 1 & 3 & - \\
\hline & Ausente & 14 & 2 & 4 & 8 & - & 11 & 2 \\
\hline \multirow[t]{2}{*}{ IV } & Presente & 15 & 10 & 2 & 2 & 1 & 5 & - \\
\hline & Ausente & 4 & - & 1 & 2 & 1 & 4 & - \\
\hline \multirow[t]{2}{*}{ Sub-totais } & Presente & 43 & 20 & 7 & 13 & 3 & $20(46,51 \%)$ & $5(11,62 \%)$ \\
\hline & Ausente & 54 & 6 & 11 & 32 & 5 & $38(70,37 \%)$ & $14(25,92 \%)$ \\
\hline Total & & 97 & 26 & 18 & 45 & 8 & 58 & 19 \\
\hline
\end{tabular}

$n=$ número de doentes.

relacionados ao tumor e aqueles relacionados ao tratamento. Os fatores relacionados aos doentes não estão ainda totalmente estudados, porém, quando investigados, têm se mostrado de grande influência na evolução, sendo, portanto, essenciais na avaliação prognostica ${ }^{34}$. Os fatores relacionados ao tumor incluem características conhecidas, tais como a classificação do tumor primário, tipo e grau histológico, comprometimento linfonodal e presença de metástases. Fatores incluindo receptores hormonais, marcadores bioquímicos, expressão de oncogenes, expressão de antígenos relacionados e, mais recentemente, marcadores moleculares do processo de carcinogênese, têm sido amplamente estudados ${ }^{35}$. Com relação ao impacto dos fatores relacionados ao tratamento instituído, é aceito que o procedimento terapêutico irá influenciar a evolução da doença, sendo particularmente verdadeiro quando se sabe que a escolha do tratamento cirúrgico constituise na primeira opção para a abordagem do CCR, acrescido do fato de que os fatores prognósticos, encontram no exame do espécime cirúrgico, extenso campo de pesquisa $^{31}$.

$\mathrm{O}$ estudo da IN como fator relacionado ao prognóstico do CCR, diferentemente da invasão vascular, não tem merecido destaque na literatura, sendo mencionada apenas como provável variável a ser considerada. Todavia, trabalhos vêm chamando atenção para a IN como potencial fator preditivo, com base no estudo realizado pela técnica da hematoxilina-eosina 25,27 . Esta técnica, pela falta de acuidade em identificar o comprometimento neural, é cada vez mais questionada ${ }^{34}$. Bellis et al. ${ }^{29}$ publicaram estudo comparativo entre a técnica da hematoxilina-eosina e a imunoistoquímica para pesquisa da expressão tecidual

Tabela 4 - Incidência da invasão neural com relação à recidiva total e resultado estatístico.

\begin{tabular}{lccc}
\hline Recidiva & Positiva & $\begin{array}{c}\text { Invasão Neural } \\
\text { Negativa }\end{array}$ & Total (\%) \\
\hline Presente & 28 & 17 & $45(46,39 \%)$ \\
Ausente & 15 & 37 & $52(53,61 \%)$ \\
Total & 43 & 54 & 97 \\
\hline
\end{tabular}

Teste $c^{2}, p=0,0010^{*}$ 
Importância Prognóstica da Invasão Neural no Câncer Colorretal:

Estudo Imunoistoquímico com a Proteína S-100

José Vinícius Cruz e Cols.
Vol. 26 da proteína S-100, analisando, sob o prisma histopatológico, a IN em espécimes de CCR. Mostraram de forma inequívoca, a superioridade da técnica imunoistoquímica para proteína S-100 sobre o método da hematoxilina-eosina na identificação da IN. Outros estudos comparando as duas técnicas, na avaliação da IN em doentes com câncer gástrico, demonstraram que a imunoistoquímica poderia ser considerada como "padrão ouro" para a identificação do comprometimento nervoso ${ }^{33}$. Tais aspectos também foram encontrados no presente estudo, constatando-se que a pesquisa da IN pela hematoxilina-eosina apresentou acurácia, sensibilidade, valor preditivo positivo e valor preditivo negativo criticáveis, tornando-a passível de exageradas faixas de erro diagnóstico. Estas observações sugerem que, quando se deseja estudar a IN no CCR com maior precisão, torna-se recomendável o emprego de técnicas imunoistoquímicas.

Tem sido demonstrado que tumores colorretais com IN possuem crescimento infiltrativo, apresentam maior possibilidade de disseminação linfonodal e encontram-se associados a estádios mais avançados da doença ${ }^{36}$. Tumores com IN também mostram maiores índices de recidiva e pior prognóstico ${ }^{37}$. Dessa maneira, a pesquisa rotineira da IN pode revelar-se um fator útil adicional na avaliação da malignidade do CCR. Contudo, poucos autores avaliaram a IN como fator prognóstico independente quanto à sobrevida e ao intervalo livre de doença no $\mathrm{CCR}^{38}$.

$\mathrm{Na}$ presente casuística, a análise univariada permitiu calcular a sobrevida (em meses), conforme as variáveis consideradas, possibilitando determinar o grau de significância estatística para cada uma delas. Para cada graduação de uma mesma variável, foi calculada a sobrevida. Verificou-se que apenas seis entre as relacionadas apresentavam importância significativa: classificação de Dukes, Astler-Coller, tipo histológico, IN estudada pela expressão tecidual da proteína $\mathrm{S}-100$, número de linfonodos comprometidos e a porcentagem de linfonodos comprometidos em relação ao número total dissecado. Assim sendo, a sobrevida média apresentou variação significativa nestas seis variáveis, segundo os testes de Cox-Mantel e Breslow.

Quando se calculou a sobrevida livre de doença (em meses), conforme as variáveis consideradas, determinando o grau de significância de cada uma delas, verificou-se que cinco apresentavam importância significativa: sexo, classificação de Dukes, Astler-Coller,
IN estudada pela expressão tecidual da proteína S-100 e a porcentagem de linfonodos comprometidos. Cabe destacar também que na análise da variável sexo, referente ao aspecto masculino, mostrou-se marginalmente significante pelo teste de Breslow, tendo ocorrido o mesmo resultado para o quesito porcentagem de linfonodos comprometidos, neste caso com a análise realizada pelo teste de Cox-Mantel.

A partir das variáveis que mostraram melhor significado prognóstico na analise univariada, foram selecionadas aquelas que serviram para realizar o modelo passo a passo, com critérios de inclusão condicional, que nortearam a realização das regressões multivariadas, para análise da sobrevida e sobrevida livre de doença. As curvas de sobrevida sempre foram relacionadas com a variável IN determinada pela pesquisa da proteína S-100. Quando a sobrevida foi relacionada à categoria Dukes $\mathrm{B}$, e a presença ou não de IN detectada pelo método da proteína S-100, verificou-se que as curvas de sobrevida foram estatisticamente diferentes, com prejuízo para os doentes com IN positiva ( $\mathrm{p}=0,0213)$. O mesmo aspecto foi observado quando se consideraram os enfermos classificados no estádio C de Dukes ( $\mathrm{p}=0,0007)$.

Quando se estudou a sobrevida livre de doença, relacionada às categorias Astler-Coller B1 e B2 contra a IN pela proteína S-100, não se observou diferença significante. No entanto, quando foram analisados os doentes nos estádios C1 e C2 de Astler-Coller, com IN positiva ou negativa, verificou-se que as curvas foram estatisticamente diferentes $(\mathrm{p}=0,0014 \mathrm{e} \mathrm{p}$ $=0,0177$, respectivamente), evidenciando evolução pior para os doentes que apresentavam IN positiva pela pesquisa da proteína S-100. Desse modo, foi possível dispor de um conjunto de informações que coloca a IN como fator relacionado ao pior prognóstico, em várias instâncias da análise, evidenciando comportamento de maior agressividade tumoral no CCR.

Examinando a literatura, observa-se evidente dificuldade na correlação e interpretação das variáveis prognósticas em relação ao câncer em geral, pela imensa gama de fatores envolvidos, relacionados ao doente, tumor e terapêutica. Assim, com objetivo de realizar análise mais lúcida entre variáveis anatomopatológicas e sobrevida de pacientes com CCR, submetidos à cirurgia com intenção curativa, Jass e Morson, em 1987, 31 criaram uma tabela de pontuação, baseada em oito diferentes variáveis anatomopatológicas submetidas à regressão multivariada. Finalmente, selecionaram quatro 
delas para, de acordo com a gradação das mesmas, formular tabelas de pontuação para posterior agrupamento dos doentes em quatro classes crescentes de gravidade. $\mathrm{O}$ agrupamento permite estudar o número de óbitos por outras causas, a sobrevida com e sem recidiva da doença e, finalmente, a sobrevida nos períodos de cinco e 10 anos. As variáveis selecionadas pelos autores foram: grau de infiltração da parede intestinal, infiltrado inflamatório peritumoral, tipo de crescimento do tumor e número de linfonodos comprometidos.

No presente estudo, acrescentou-se à classificação proposta por Jass e Morson ${ }^{31}$, a IN estudada pelo método imunoistoquímico para pesquisa da expressão tecidual da proteína S-100, dividindo os enfermos em dois grupos baseados na presença ou ausência de comprometimento nervoso. Ao analisar-se a sobrevida em cinco e 10 anos nos doentes com e sem IN foi possível verificar que, não obstante os doentes com IN positiva estivessem enquadrados em uma classe da classificação de Jass e Morson ${ }^{31}$ de menor gravidade (Classe I), ao final de 10 anos, dos 16 enfermos com IN positiva só cinco permaneciam vivos, enquanto dos 26 enfermos em que não havia IN, 10 permaneciam vivos. Nas outras três classes não existia um doente sequer com IN positiva que permanecesse vivo após cinco anos de seguimento. Ao analisar-se o grupo de pacientes em que não existia IN, avaliada pela expressão tecidual da proteína S-100, foi possível alcançar sobrevida em 10 anos, se bem que em pequeno número de doentes, tanto na classe II quanto na classe III. Cabe ressaltar que no grupo em que não existia IN, 14 enfermos pertencentes às classes I, II e III, apresentaram sobrevida próxima aos 10 anos de seguimento.

Estabelecendo-se igual raciocínio para pacientes com IN, agrupados nas classes II e III, observaram-se oito casos de sobrevida em cinco anos, enquanto que, na classe III sem IN neoplásica, encontraram-se 19 doentes com sobrevida alcançada de cinco anos. Como conseqüência, as classes constituídas de pacientes sem IN poderiam enriquecer o número de casos com sobrevida de até 10 anos. Estas conclusões não puderam ser estendidas para os doentes com IN.

Finalmente, ao se concentrar a atenção no subtotal de sobrevida com cinco e 10 anos nos diferentes casos com e sem IN, verifica-se que, enquanto $46 \%$ dos doentes (20/43) com IN alcançaram sobrevida de cinco anos, o mesmo período foi atingido por $70 \%$ dos enfermos (38/54) sem infiltração nervosa. Quando se considera prazo de 10 anos de sobrevida, as porcentagens são 11\% (5/43) e 25\% (14/54), respectivamente, para os doentes com e sem IN.

Considerando as variáveis que mostraram melhor prognóstico, selecionadas pelos testes de CoxMantel e Breslow, foi possível separar dois conjuntos diferentes, para serem submetidos a analise multivariada de fatores prognósticos, para sobrevida e sobrevida livre de doença.

As variáveis incluídas na análise multivariada para a sobrevida foram: classificação de Dukes, AstlerColler, IN pesquisada pela proteína S-100, número de linfonodos, porcentagem de linfonodos comprometidos pelo tumor e, finalmente o tipo do tumor. A análise multivariada no que concerne à sobrevida demonstrou que a classificação de Dukes e a IN pesquisada pela proteína S-100 constituíam-se fatores prognósticos independentes.

$\mathrm{Na}$ análise multivariada de fatores prognósticos para a sobrevida livre de doença, utilizando-se os mesmos critérios, selecionaram-se as seguintes variáveis que se mostraram mais favoráveis na análise univariada: sexo, classificação de Dukes, Astler-Coller, porcentagem de linfonodos comprometidos, tipo de tumor e IN pesquisada pela proteína S-100. Verificou-se que a variável sexo apresentou resultado com diferença desfavorável para o sexo feminino, porém tal resultado não é comparável à literatura. Com relação à classificação de Astler-Coller, constatou-se que essa variável demonstrou significância estatística e que o grupo de doentes classificados como $\mathrm{C} 2$, contribuiu para influenciar o resultado da análise multivariada. Quanto à IN pesquisada pela expressão tecidual da proteína S100 , a regressão multivariada também demonstrou tratar-se de fator prognóstico independente para a sobrevida livre da doença.

Ao analisar-se a relação entre recidiva neoplásica e a IN, dos 97 doentes portadores de CCR, operados com intenção curativa, foi possível observar que a doença recidivou em 46,39\% (45/97) dos casos, sendo que em 19 sob o tipo loco-regional e nos 26 restantes como metástases à distância. A análise da presença ou ausência de recidiva tumoral como um todo, contra a IN, constata que este tipo de cruzamento de variáveis apresenta diferença, estatisticamente significante $(\mathrm{p}=0,0010)$. Tal achado é facilmente compreendido, pelo comportamento 
Importância Prognóstica da Invasão Neural no Câncer Colorretal:

Estudo Imunoistoquímico com a Proteína S-100

José Vinícius Cruz e Cols.
Vol. 26 diametralmente oposto quanto à incidência de recidivas de acordo com a presença ou não de IN pelo tumor. Assim, quando ocorreu recidiva tumoral quase $2 / 3$ destes pacientes (28/45) apresentavam IN, enquanto que dos 52 casos que não apresentavam recidiva, menos de 1/3 destes (15/52) tinham infiltração nervosa pela neoplasia, situação esta confirmada pelo estudo estatístico.

Dos 45 casos com recidiva, 26 foram a óbito (18 por metástases à distância e oito por recidiva locoregional). Dos 19 casos restantes com recidiva da doença, ou seja, pacientes vivos com evidência da neoplasia, 11 apresentaram recidiva loco-regional e oito metástases à distância. Cabe destacar que o acompanhamento pós-operatório mostrou que, no primeiro ano após a intervenção cirúrgica inicial, todos os sete casos com recidivas eram portadores de IN pelo tumor. Entre o primeiro e segundo ano pós-operatório, dos 13 casos com recidiva, 8 apresentavam infiltração nervosa. Portanto, dos 20 enfermos que apresentaram recidiva neoplásica nos dois primeiros anos de seguimento $75 \%$ (15/20) possuíam comprometimento neural.

Ao analisarem-se os óbitos por recidiva tumoral, constata-se que nos dois primeiros anos de seguimento ocorreram 16 mortes, sendo 13 em enfermos com IN. Dos 16 óbitos, 10 por metástases à distância das quais em apenas um caso não havia IN. Quando se analisam os 9 enfermos com recidiva neoplásica, ocorrida no terceiro ano pós-operatório, verificou-se que elas apresentavam maior prevalência (6/9) nos doentes com IN detectada. Na análise da sobrevida a partir do quarto ano pós-operatório, constatou-se que passou a existir predomínio dos enfermos sem IN, havendo, num total de nove doentes, seis que não apresentavam comprometimento neural. Dos quatro óbitos ocorridos no período, três ocorreram por metástases à distância e apenas um por recidiva locoregional. A partir do quarto ano pós-operatório, houve equilíbrio entre a incidência de casos com e sem IN, mas continuou predominando o número de enfermos com metástases à distância, como forma de recidiva da doença, o que ocorreu em cinco casos de um total de sete.

Estes achados demonstram que a recidiva da doença foi mais precoce e relacionada a maior número de mortes nos enfermos que apresentavam IN. A partir dos resultados do presente estudo, pode-se inferir que, na condição clínica de recidiva neoplásica, nos enfermos que apresentam IN, os tumores demonstraram comportamento mais agressivo por reaparecerem mais precocemente e encontrarem-se associados a maiores índices de mortalidade.

Novas informações esclarecendo os mecanismos genéticos envolvidos na carcinogênese do CCR vêm sendo obtidas, com objetivo de propor terapias neoadjuvantes, cirúrgicas ou adjuvantes, visando melhorar a sobrevida dos doentes. Com a melhor compreensão do papel desempenhado pelo maior número de genes envolvidos na carcinogênese, por meio de técnicas de microarrays, é possível que se esteja entrando na era do estadiamento molecular do CCR. Recentes estudos comparando o potencial de um grupo de genes em sobreviventes e não sobreviventes portadores de CCR, com a efetividade de predição prognóstica da classificação de Dukes, demonstraram resultados significativamente melhores para o estadiamento genético ${ }^{39}$. Contudo, são técnicas onerosas, de difícil disponibilidade e ainda pouco empregadas na prática clínica.

Apesar de todo o avanço conquistado nos últimos anos, ainda não se conseguiu modificar a realidade bidirecional em que vivem os doentes com CCR: aqueles que esperam viver e os destinados a morrer. Somente com conhecimento do maior número de variáveis relacionadas ao prognóstico do CCR, fidedignas e passíveis de serem analisadas com metodologia exeqüível e, se possível, de baixo custo, poderão mudar-se os resultados atuais. Os resultados do presente estudo permitem situar a análise imunoistoquímica da proteína S-100 na avaliação da IN em portadores de CCR como uma metodologia que atende a esses predicados.

\section{CONCLUSÕES}

Nas condições do presente estudo, pode-se concluir que o método da hematoxilina-eosina apresenta fraca habilidade na identificação da IN. A IN demonstrou ser fator prognóstico independente nos doentes portadores de CCR. A IN no CCR, detectada pela pesquisa imunoistoquímica da proteína S-100, acrescenta informação prognóstica quanto à sobrevida nos doentes classificados nos estádios B e C de Dukes e sobrevida livre de doença nos doentes classificados nos estádios C1 e C2 da classificação de Astler-Coller. 
Rev bras Coloproct Julho/Setembro, 2006
Importância Prognóstica da Invasão Neural no Câncer Colorretal:

Estudo Imunoistoquímico com a Proteína S-100

José Vinícius Cruz e Cols.
Vol. 26

ABSTRACT: Among the anatomopathological variables related to the prognosis for patients with colorectal cancer, neural invasion remains little studied. Objective: To evaluate whether neural invasion in patients with colorectal cancer in Dukes stages $B$ and $C$ could be considered to be an independent prognostic factor, by means of univariate and multivariate analysis. Method: Ninety-seven patients who underwent operations with curative intent by the same surgical team were followed up for a minimum period of five years and were studied. Patients who received adjuvant treatment were excluded. The surgical specimens were stained with hematoxylin-eosin (HE) and immunohistochemical techniques for S-100 protein analysis, with the aim of comparing the two techniques for detecting neural invasion. Accuracy, specificity, sensitivity and positive and negative predictive values were analyzed for $\mathrm{HE}$ in relation to $\mathrm{S}-100$ protein. Comparison between the incidence of neural invasion and tumor recurrence was made by using the chi-squared test. Survival and disease-free survival were studied by univariate analysis. A significance level of $5 \%(p £ 0.05$ ) was established for all the tests adopted. Results: The HE technique presented weak ability to detect neural invasion and was inadequate for this analysis in colorectal cancer patients. The survival and disease-free survival curves for patients with neural invasion, investigated by means of immunostaining for S-100 protein, were significantly worse, thus identifying this histological characteristic as having independent prognostic value $(p=0.0003$ and $p=0.0002$, respectively). There was significantly more tumor recurrence among patients who presented neural invasion $(p=0.0010)$. Conclusion: The results from the present study allow the conclusion that, among colorectal cancer patients, neural invasion was shown to be an independent variable that gave additional prognostic information regarding patients in stages B, C and C2 of the Dukes and Astler-Coller classifications, respectively.

Key words: Neoplasms. Colorectal Neoplasms. S 100 Protein. Immunohistochemistry. Prognosis.

\section{REFERENCIAS}

1. Boring CC, Squires TS, Tong T. Cancer statistics 1993. CA Cancer J Clin 1993; 43:7-26.

2. Garfinkel L, Mushinski M. U.S. cancer incidence, mortality and survival: 1973-1996. Stat Bull Metrop Insur Co. 1999; 80:23-32.

3. Instituto Nacional do Câncer. Estimativas da incidência e mortalidade por câncer no Brasil 2003. Disponível em http:// www.inca.gov.br. (Acessado em 9/ jul/2004).

4. Neves FJ, Mattos IE, Koifman RJ. Mortalidade por câncer de cólon e reto nas capitais brasileiras no período 1980-1997. Arq Gastroenterol 2005; 42: 63-70.

5. Rich T, Gunderson LL, Lew R, Galdibini JJ, Cohen AM, Donaldson G. Patterns of recurrence of rectal cancer after potentially curative surgery. Cancer 1983; 52:1317-29.

6. Michelassi F, Vanucci L, Ayala JJ, Chappel R, Goldrerg R, Block GE. Local recurrence after curative resection of colorectal adenocarcinoma. Surgery 1990; 108:787-93.

7. Kemeny N, Braun DW Jr. Prognostic factors in advanced colorectal carcinoma. Importance of lactic dehydrogenase level, performance status, and white blood cell count. Am J Med 1983; 74:786-96.

8. Jass JR, Mukawa K, Goh HS, Love SB, Capellaro D. Clinical importance of DNA content in rectal cancer measured by flow cytometry. J Clin Pathol 1989; 42:254-9.

9. Ponz de Leon M, Sant M, Micheli A, Sacchetti C, Di Gregorio $\mathrm{C}$, Fante R, et al. Clinical and pathologic prognostic indicators in colorectal cancer. A population-based study. Cancer 1992; 69:626-35.

10. Sanfelippo PM, Beahrs OH. Factors in the prognosis of adenocarcinoma of the colon and rectum. Arch Surg 1972; 104:401-6.
11. Cain AS, Longino LA. Carcinoma of the colon in children. $\mathbf{J}$ Pediatr Surg 1970; 5:527-32.

12. Wolmark N, Wieand HS, Rockette HE, Fischer B, Glass A, Lawrence $\mathrm{W}$, et al. The prognostic significance of tumor location and bowel obstruction in Dukes B and C colorectal cancer. Findings from the NSABP clinical trials. Ann Surg 1983; 198(6):743-52.

13. Grinnel RS. The grading and prognosis of carcinoma of the colon and rectum. Ann Surg 1939; 109:500-33.

14. Dukes CE. The classification of cancer of the rectum. J Pathol 1932; 35:323-32.

15. Astler VB, Coller FA. The prognostic significance of direct extension of carcinoma of the colon and rectum. Ann Surg 1954; 139:846-51.

16. .Dukes CE, Bussey HJR. The spread of rectal cancer and its effect on prognosis. Br J Cancer 1958; 12:309-20.

17. Halvorsen TB, Sein E. Influence of mucinous components on survival in colorectal adenocarcinomas: a multivariate analysis. J Clin Pathol 1988; 41:1068-72.

18. Cutait R. Contribuição ao estudo do antígeno carcinoembrionário e dos antígenos de superfície ABH no câncer colo-retal. [Tese]. São Paulo: Faculdade de Medicina da Universidade de São Paulo. 1991.

19. Martinez CAR, Priolli DG, Cardinalli IA, Piovesam H, Pereira JA. Importância da correlação entre o padrão de distribuição tecidual do antígeno carcinoembriônico e seus níveis séricos no prognóstico do câncer colorretal. Estudo prospectivo de 50 casos. Rev Bras Coloproct. 2004; 24 supl 1: 90 .

20. Khankhanian N, Mavligit GM, Russell WO, Schimek M. Prognostic significance of vascular invasion in colorectal cancer of Dukes’ B class. Cancer 1977; 39:1195-200. 
21. Knudsen JB, Nilsson T, Sprechler M, Johansen A, Christensen $\mathrm{N}$. Venous and nerve invasion as prognostic factors in postoperative survival of patients with resectable cancer of the rectum. Dis Colon Rectum 1983; 26:613-7.

22. Phillips RK, Hittinger R, Blesowsky L, Fry JS, Fielding LP. Large bowel cancer: surgical pathology and its relationship to survival. Br J Surg 1984; 71:604-10.

23. Kune GA, Kune S, Field B, White R, Brough W, Schellemberger $\mathrm{R}$, et al. Survival in patients with large-bowel cancer. A population-based investigation from Melbourne Colorectal Cancer Study. Dis Colon Rectum 1990; 33:938-46.

24. Pinho MSL. Estadiamento molecular do câncer colorretal: O futuro se aproxima. Rev bras Coloproct 2005; 25:279-84.

25. Krasna MJ, Flancbaum L, Cód RP, Shneibaum S, Ari GB. Vascular and neural invasion in colorectal carcinoma: incidence and prognostic significance. Cancer 1988; 61:1018-23.

26. Horn A, Dahl O, Morild I. Venous and neural invasion as predictors of recurrence in rectal adenocarcinoma. Dis Colon Rectum 1991; 34:798-804.

27. Shirozu K, Hiroharu I, Kakegawa T. Prognostic evaluation of perineural invasion in rectal cancer. Am J Surg 1993; 165:233-7.

28. Jass JR. Lymphocitic infiltration and survival in rectal cancer. J Clin Pathol 1986; 39:585-9.

29. Bellis D, Marci V, Monga G. Light microscopic and immunohistochemical evaluation of vascular and neural invasion in colorectal cancer. Pathol Res Pract.1993; 189:443-8.

30. Mori M, Adachi Y, Kamakura T, Ikeda Y, Maehara Y, Sugimachi K. Neural invasion in gastric carcinoma. J Clin Pathol.1995; 48:137-42.
31. Jass JR, Morson BC. Reporting colorectal cancer. J Clin Pathol.1987; 40:1016.

32. .Simon R. Importance of prognostic factors in cancer clinical trials. Cancer Reserch 1984; 68:185-92.

33. Sather HN. The use of prognostic factors in clinical trials. Cancer. 1986; 58:461-7.

34. Feinstein AR. On classifying cancers while treating patients. Arch Intern Med 1985; 145:1789-91.

35. Fielding LP, Fenoglio-Preiser CM, Freedman LS. The future of prognostic factors in outcome prediction for patients with cancer. Cancer.1992; 70:2367-77.

36. Matsushima T, Mori M, Kido A, Adachi Y, Sugimachi K. Preoperative estimation of neural invasion in rectal carcinoma. Oncol Rep.1998; 5:73-6.

37. Durante AP, Bromberg SH, Barreto E, Capellano G, Godoy AC. Prognostic value of lymphatic vessel and neural invasion in colorectal carcinoma. Rev Ass Med Bras 2004; 50:21-6.

38. Bognel C, Rekacewicz C, Mankarios H, Pignon JP, Elias D, Duvillard P, et al. Prognostic value of neural invasion in rectal carcinoma: a multivariate analysis of 339 patients with curative resection. Eur J Cancer 1995;31:894-8.

39. Eschrich S, Yang I, Bloom G, Kwong KI, Boulware D, Cantor A, et al. Molecular staging for survival prediction of colorectal cancer patients. J Clin Oncol 2005; 23:3526-35.

\section{Endereço para Correspondência:}

\section{CARLOS AUGUSTO REAL MARTINEZ}

Rua Rui Barbosa, 255 apto 32

09.190-370 - Santo André (SP)

Tel. (11) 4438-9203 